\title{
An FFT-based algorithm for reconstructing inhomogeneous circular cylindrical shells from noisy data
}

\author{
S JEGANNATHAN and BHASKAR RAMAMURTHI \\ Department of Electrical Engineering, Indian Institute of Technology, \\ Madras 600036, India
}

\begin{abstract}
The inverse problem of reconstructing an inhomogeneous, circular, cylindrical shell, from the knowledge of scattering data, is of importance in diverse fields, including the imaging of thin veins and arteries in medicine, of co-axial cables in non-destructive testing, and of volcanic pipes in volcanology. Optical fibres provide another important example of shells evaluated by such non-invasive means.

In as much as the problem has such diverse applications, it will be useful to have an algorithm for this purpose, which (i) is computationally efficient; (ii) provides good reconstructions when the data is corrupted with additive noise which is often assumed to be Gaussian; (iii) does not employ a small-perturbations approximation, such as Born's or Rytov's, which restricts their scope of application.

One such algorithm is proposed in this paper. Here, computational efficiency is achieved by recognising that the circular geometry of the problem translates itself into circulant matrices that are easily inverted by invoking the Fast Fourier Transform (FFT); while the desensitization of the method to corrupting noise is carried out by employing the leastsquares method.
\end{abstract}

Illustrative computer simulations verify the validity of the technique proposed.

Keywords. Reconstruction; imaging; inverse problem; cylindrical shells.

\section{Introduction}

The inverse problem of reconstructing a cylindrical shell, from the knowledge of scattering data, is of importance to researchers in several fields. Some important examples include the imaging of thin veins and arteries in medicine, of co-axial cables in non-destructive testing, and of volcanic pipes in volcanology. Optical fibres provide another important example of shells evaluated by such non-invasive means.

Several publications on this problem have appeared in the literature during the recent years. Boerner et al (1971) has proposed an exact method for imaging a circular cylinder, which is a special case of a shell having zero inner radius, but the method can be applied only when the scatterer is a perfect conductor. Marcuse (1975) and several other authors (Presby \& Marcuse 1974; Watkins 1974; Holoubek 1976) have 
considered the characterization of optical fibres, while Duchene \& Tabara (1983) treat the blood-vessel-assessment problem in great detail, all based on the geometricaloptics approximation. However, the former techniques are generally useful only when the diameter of the shell is much larger than the illuminating wavelength, while the latter are of use only for imaging homogeneous shells. The homogeneous shell problem has also been considered by Datta \& Som (1981). An approach based on the Gelfand-Levitan theory is furnished by Smith (1987), but this approach cannot handle angular variations of inhomogeneities, as it has been specifically tailored for the radially-varying case.

Thus, it appears that there exists no special technique to solve the inverse problem of a thin, angularly inhomogeneous, strongly-scattering shell and we have to take recourse to diffraction tomographic techniques. The literature on diffraction tomography is vast (Haykin 1985) encompassing a variety of algorithms that exhibit a great deal of diversity in their scope of applications. Care, therefore, must be exercised in selecting an algorithm that is pertinent to the problem under consideration and tailoring it to the specific demands. For instance, many an algorithm incorporates a small-perturbational assumption such as Born's or Rytov's (Schueler et al 1984). While these elegant solutions are invaluable for imaging a weak-scatterer, and testing algorithms meant for strong scatterers in the weak-scattering limit, they are not suitable for imaging a strong scatterer as such.

A tomographic scheme recently proposed by the authors (Jegannathan \& Ramamurthi 1990b) seems to be an appropriate tool to solve this problem. This method does not make any small-perturbation assumption, and uses the fast Fourier transform to arrive at the solution efficiently. However, the availability of ideal measurements' data is presumed in Jegannathan \& Ramamurthi (1990b), and the algorithm is pathologically sensitive to any deviations therefrom. Since such ideal data will never be available due to the presence of errors including (i) measurement errors, (ii) errors due to the dynamic range limitations of transducers and other measuring instruments, (iii) quantization errors, (iv) discretization errors, and (v) modelling errors, the proposed method cannot be utilised in practice. In this paper, this algorithm is modified so that it works even with noisy data when the scatterer happens to be a shell.

An alternative to the approach presented here is the sinc-basis multiple-source moment-method pioneered by Tracey, Johnson and other (Tracey \& Johnson 1983; Johnson et al 1984; Cavicchi et al 1988). Their method may provide greater flexibility in choosing the measurement points compared to the approach presented here, although at the cost of more complex calculations.

Jegannathan \& Ramamurthi (1990b) have formulated the problem in terms of a system of linear equations of the form $\mathbf{A x}=\mathbf{y}$. A problem characterised by $\mathbf{A x}=\mathbf{y}$ is said to be ill-posed, if any one of the following conditions, known as Hadamard's conditions (proposed as early as 1900!), are violated.

(i) the solution $\mathbf{x}$ exists for each element $\mathbf{y}$ in the range space $Y$;

(ii) the solution $\mathbf{x}$ is unique;

(iii) small perturbations in $\mathbf{y}$ result in small perturbations in the solution $\mathbf{x}$ without the need to impose additional constraints.

When a problem is ill-posed, an attempt must be made to regularize it (Nashed 1976) so that the solution to the regularized version is a reasonable approximation of the actual solution. This can be achieved by (Nashed 1976; Sarkar et al 1981) 
(i) changing the definition of what is meant by an acceptable solution;

(ii) changing the space to which the acceptable solution belongs;

(iii) revising the problem statement;

(iv) introducing regularizing operators; and

(v) introducing probabilistic concepts so as to obtain a stochastic extension of the original deterministic problem.

In the following sections it will be shown that the algorithm specified in Jegannathan \& Ramamurthi (1990b), as applied to the shell problem, is ill-posed because it violates the third of Hadamard's conditions, and can be regularized by a combination of the first and third techniques specified above, the regularization being done in a way such that the computational advantages of the parent algorithm, are not sacrificed.

\section{Basic theory}

Consider a harmonic wave incident in free space on a dielectric shell as suggested in figure 1. The time-factor $e^{i \omega t}$ is understood. It is assumed that the incident electric field intensity and the inhomogeneities are not functions of $z$, where the $z$-axis is taken to be parallel to the axis of the cylindrical shell.

The scattering pattern of the shell is determined by dividing it into $N$ identical cells, sufficiently small so that the dielectric constant and the total electric field intensity are essentially constant over each cell, as indicated in figure 1 . The total electric field is treated as an unknown which is determined by solving a system of linear equations of the form (Richmond 1965):

$$
(\mathbf{I}+\mathbf{K} \tilde{\varepsilon}) \mathbf{e}=\mathbf{e}^{\mathbf{j}}
$$

where $e$ and $e^{i}$ are column vectors with elements $\left\{e_{n}\right\}$ and $\left\{e_{n}^{i}\right\}, n=1,2, \ldots N, I$ is an $N \times N$ unity matrix and $\tilde{\varepsilon}$ is a diagonal matrix whose element in the nth row and column is given by $\varepsilon_{n}-1$. Here, $e_{n}, e_{n}^{i}$ and $\varepsilon_{n}$ denote the total and incident field intensities and the average dielectric constant over cell $n$, respectively, while $K$ is an $N \times N$ matrix whose elements $\left\{k_{m n}\right\}$ depend on the distance between the centres of cell $m$ and $n$.

Once the system of linear equations (1) has been solved, the scattered field can be

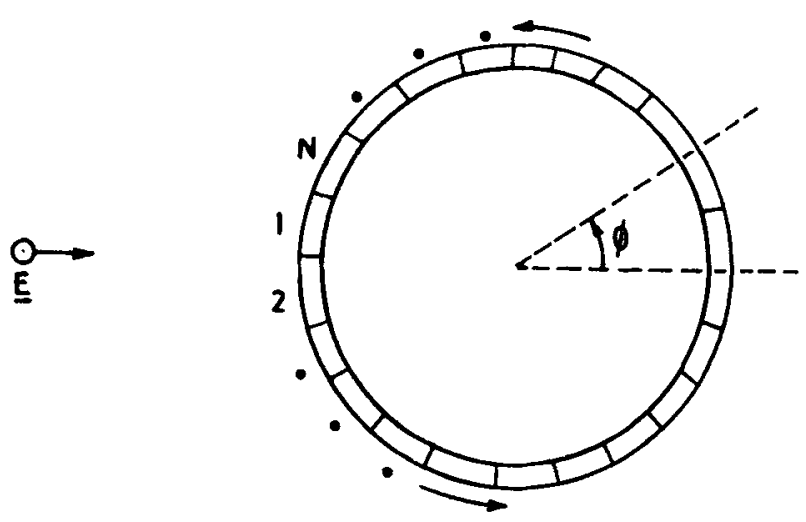

Figure 1. Illustrates the division of a dielectric cylindrical shell into $N$ identical cells. 
determined at any point in space by means of a simple calculation as described in Richmond (1965). Specifically, the column vector $\tilde{\mathbf{e}}^{\mathrm{s}}$ with elements $\left\{\tilde{e}^{s}\right\}$, where $\tilde{e}^{s}$ is the value of the scattered field at a measurement point outside the scatterer with coordinates $\left(x_{m}, y_{m}\right) m=1,2, \ldots, N$, is given by

$$
\tilde{\mathbf{e}} \mathbf{s}=\mathbf{A} \tilde{\varepsilon} \mathbf{e} \text {. }
$$

Here, $\mathrm{A}$ is an $N \times N$ matrix with elements $\left\{a_{m n}\right\}$ proportional to the distance $\rho_{m n}$ between the $m$ th measurement point and the $n$th cell centre.

Now, the inverse problem is to find $\tilde{\varepsilon}$ by solving (1) and (2), given $\tilde{e}^{s}$ and $\mathbf{e}^{\mathbf{i}}$, and the coefficient matrices $\mathbf{A}$ and $\mathbf{K}$. This can be done by the following algorithm.

\section{Algorithm 1.}

(i) Solve for the product $\tilde{\varepsilon}$ from (2).

(ii) Substitute $\tilde{e}$ in (1) and solve for $e$.

(iii) Divide $\tilde{\varepsilon} e$ by e to obtain $\tilde{\varepsilon}$.

Since the matrix $\mathbf{A}$ is usually large and non-sparse, the first step of algorithm 1 requires prohibitively large computational resources if it is solved using a direct procedure such as Gaussian elimination. However, as we shall show here, if the measurement points are chosen appropriately, $\mathbf{A}$ turns out to be a circulant matrix, which can be inverted very efficiently using the Fast Fourier Transform (FFT), with a computational complexity of $O(N \log N)$. The second step of the algorithm can again be implemented efficiently because of the circulant nature of $K$, as shown in Jegannathan \& Ramamurthi (1990a).

In order to see the circulant nature of $\mathbf{A}$, consider $N$ measurement points such that they all lie along a circle of radius $R$, angularly equispaced as shown in figure 2 . The polar coordinate of the $m$ th measurement point will be of the form $[R, \alpha+2 \pi m / N]$ where $\alpha$ is a constant, just as the polar coordinates of the centre of the $n$th cell will be given by $[r, \beta+2 \pi n / N], \beta$ being another arbitrary constant. Thus,

$$
\rho_{m n}=\left\{R^{2}+r^{2}-2 R r \cos [(\alpha-\beta)+(2 \pi / N)(m-n)]\right\}^{\frac{1}{2}},
$$

where $\rho_{m n}$ is as defined earlier. We can write from the above equation.

$$
\begin{aligned}
\rho_{m n} & =\rho_{m-n} \text { for } m>n ; \\
& =\rho_{m-n+N} \text { for } n \geqslant m .
\end{aligned}
$$

As mentioned earlier, the elements $\left\{a_{m n}\right\}$ of the matrix $\mathbf{A}$ are functions of the distances $\rho_{m n}$ and are of the form $f\left(\rho_{m n}\right)$. Thus, from (4), the matrix $\mathbf{A}$ can be deduced to be of the form

$$
\mathbf{A}=\left(\begin{array}{cccc}
f\left(\rho_{1}\right) & f\left(\rho_{N}\right) & \cdots & f\left(\rho_{2}\right) \\
f\left(\rho_{2}\right) & f\left(\rho_{1}\right) & \cdots & f\left(\rho_{3}\right) \\
\vdots & \vdots & \vdots & \vdots \\
f\left(\rho_{N}\right) & f\left(\rho_{N-1}\right) & \cdots & f\left(\rho_{1}\right)
\end{array}\right)
$$

showing that $\mathbf{A}$ is a circulant matrix.

It is to be noted here that the matrix $\mathbf{A}$ is circulant irrespective of the actual locations of the measurement points so long as they are spread equi-angularly, as the above analysis includes an arbitrary constant $\alpha$. 


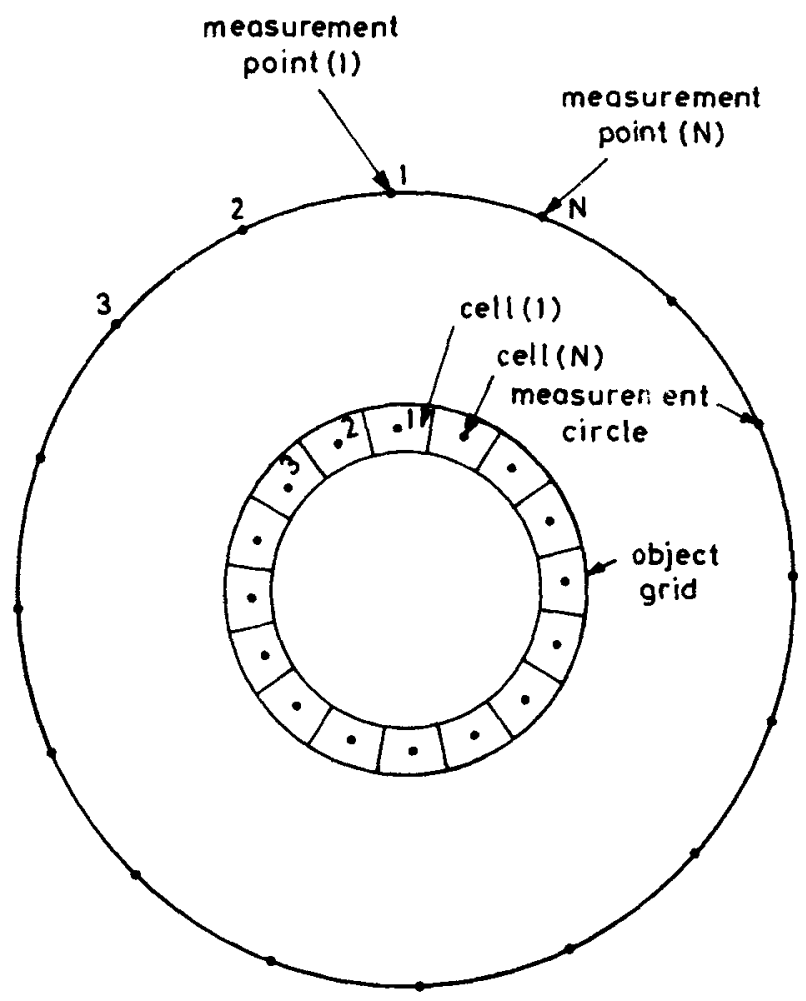

Figure 2. Measurement configuration.

If the scatterer is a shell which is not so thin, then $\mathbf{A}$ will turn out to be a block circulant matrix as indicated in Jegannathan \& Ramamurthi (1990b) of which the above analysis is an important special case.

\section{A measure for sensitivity to noise}

In the previous section, we have shown that in order to solve the shell problem, we require the solution of a set of simultaneous linear equations of the form $\mathbf{A x}=\mathbf{y}$, where the coefficient matrix A turns out to be circulant. We can follow Wilkinson (1983, pp. 189-190) to analyse the sensitivity of the problem. Specifically, if $\mathbf{y}$ in

$$
\mathbf{A x}=\mathbf{y},
$$

is changed to $\mathbf{y}+\mathbf{k}$, and if

$$
\mathbf{A}(\mathbf{x}+\mathbf{h})=\mathbf{y}+\mathbf{k}
$$

we have from (6) and (7),

$$
\mathbf{h}=\mathbf{A}^{-1} \mathbf{k}
$$

and taking a suitable norm on both sides, we have

$$
\|\mathbf{h}\| \leqslant\left\|\mathbf{A}^{-1}\right\|\|\mathbf{k}\| .
$$


Throughout this paper, we make use of the first norm (or sup-norm) which is defined as (Wilkinson 1983, pp. 56-57)

$$
\|\mathbf{A}\|_{1}=\text { maximum of } \sum_{i}\left|a_{i j}\right|
$$

where $a_{i j}$ is the element belonging to the $i$ th row and the $j$ th column.

It will be more relevant to talk about relative errors, and for this we note from (6)

$$
\|\mathbf{x}\| \geqslant\|\mathbf{y}\|\|\mathbf{A}\|^{-1} \text {. }
$$

From inequalities (10) and (11), we have

$$
\frac{\|h\|}{\|\mathbf{x}\|} \leqslant\left\|\mathbf{A}^{-1}\right\|\|\mathbf{A}\| \frac{\|\mathbf{k}\|}{\|\mathbf{y}\|} .
$$

We shall define the condition number $\kappa(\mathbf{A})$

$$
\kappa(\mathbf{A})=\left\|\mathbf{A}^{-1}\right\|\|\mathbf{A}\| \text {. }
$$

From (12) and (13), we have

$$
\frac{\|\mathbf{h}\|}{\|\mathbf{x}\|} \leqslant \kappa(\mathbf{A}) \frac{\|\mathbf{k}\|}{\|\mathbf{y}\|} .
$$

Inequality (14) says that the relative error of the solution is bounded by the relative error of the RHS times the condition number. If $\kappa(\mathbf{A})$ is near unity, it is clear that small perturbations in the RHS would induce only small perturbations in the solution. The larger the condition number, the more sensitive is the problem to noise.

It should be borne in mind that (14) is a pessimistic error bound and the actual errors may be much less than specified by (14). However, there will always be some situation for which (14) is attained.

\section{Regularization}

Let us now consider a typical shell problem. Consider a shell whose relative permittivity varies as shown in figure 3 . In table 1 , a comparison is made between the reconstruction obtained by processing ideal data' and data corrupted with $1 \%$ Gaussian noise. Here, the scattering data were derived by using the approach given in Jegannathan \& Ramamurthi (1990a), while algorithm 1 in conjunction with the FFT was employed for the reconstructions. It is evident from table 1 that all reconstructions save those corresponding to the column 'ideal data' are merely garbage. Thus, small changes in the data have produced large deviations in the solution violating Hadamard's third condition, and the problem is ill-posed.

Let us try to regularize the problem simply by revising the problem statement. $A$ simple ruse is to utilise the freedom in selecting the radius $R$. In theory, when the exact field in the angular interval $[0,2 \pi]$ is known, one circle is as good as another, and we can make use of the convenient far-field. Determination of the far-field is tantamount to the determination of the radiation pattern, from which the field over the entire space outside the scatterer can be derived (Devaney 1979). However, when 

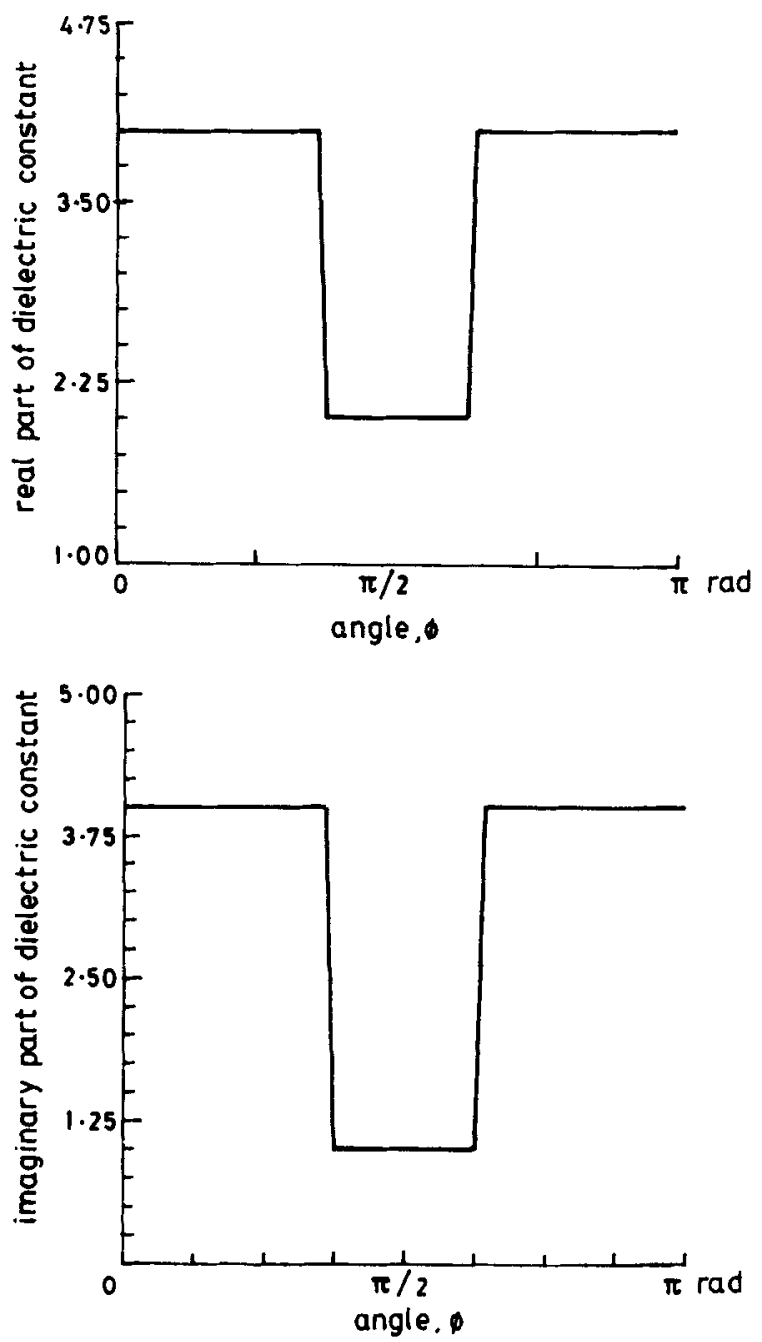

Figure 3. Depicted here is the variation of dielectric constant for $0 \leqslant \phi \leqslant \pi$, ( $\phi$ is shown in figure 1) for the shell under consideration. For simplicity, symmetry is assumed so that dielectric constant at $\phi=$ dielectric constant at $2 \pi-\phi$. The other parameters are: radius $=3.0 \lambda$; shell thickness $=0.01 \lambda$; permeability $\mu=\mu_{0}$; number of cells $N=128$.

Table 1. The drastic effect of noise. Entries denote the real and imaginary parts of the dielectric constant at selected angular positions. (Measurement radius $=4 \cdot 00 \lambda$ ).

\begin{tabular}{lcccc}
\hline Angle $\varphi$ & \multicolumn{2}{c}{ Reconstructed real parts } & & \multicolumn{2}{c}{ Reconstructed imaginary parts } \\
\cline { 2 - 3 }$=(n-1)(2 \pi / 128) n$ & With ideal data & With $1 \%$ noise & With ideal data & With $1 \%$ noise \\
\hline 1 & 4.0 & 90.3 & 4.0 & -0.775 \\
9 & 4.0 & 89.4 & 4.0 & 0.036 \\
17 & 4.0 & 89.8 & 4.0 & 0.067 \\
25 & 2.0 & 89.9 & 1.0 & -0.080 \\
33 & 2.0 & 89.7 & 1.0 & -0.540 \\
41 & 2.0 & 89.3 & 1.0 & -0.058 \\
49 & 4.0 & 92.5 & 4.0 & 1.038 \\
57 & 4.0 & 89.7 & 4.0 & -0.283 \\
65 & 4.0 & 89.9 & 4.0 & -0.046 \\
\hline
\end{tabular}


Table 2. The increase of the sup-norm based condition number (or the first norm) with the measurement radius $R$. The lower this condition number, the less sensitive the solution is to noise.

\begin{tabular}{lllccc}
\hline $\begin{array}{c}\text { Measurement } \\
\text { radius, } R \text { (in } \lambda)\end{array}$ & 10.0 & 60 & 5.0 & 4.0 & 3.01 \\
$\begin{array}{c}\text { Sup-norm based } \\
\text { condition number }\end{array}$ & $7.8 \times 10^{18}$ & $1.6 \times 10^{18}$ & $1.6 \times 10^{14}$ & $5.1 \times 10^{8}$ & $2.9 \times 10^{2}$ \\
\hline
\end{tabular}

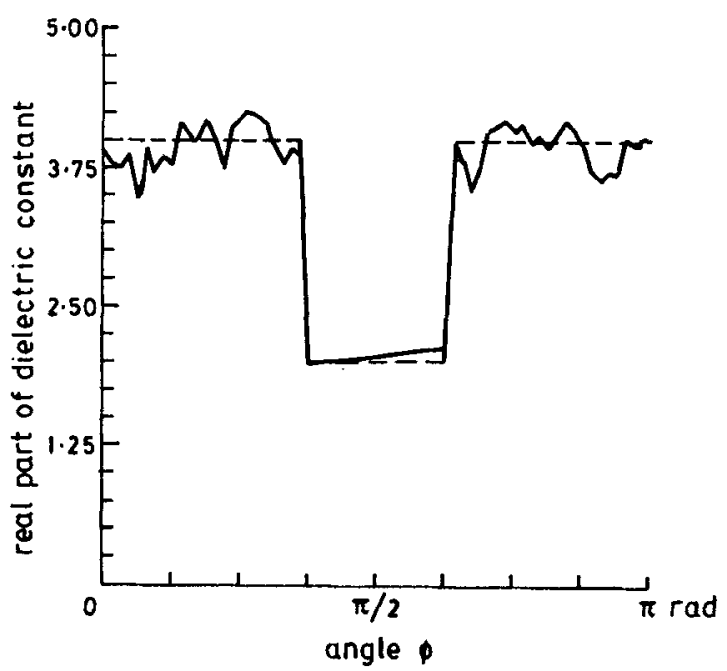

(a)

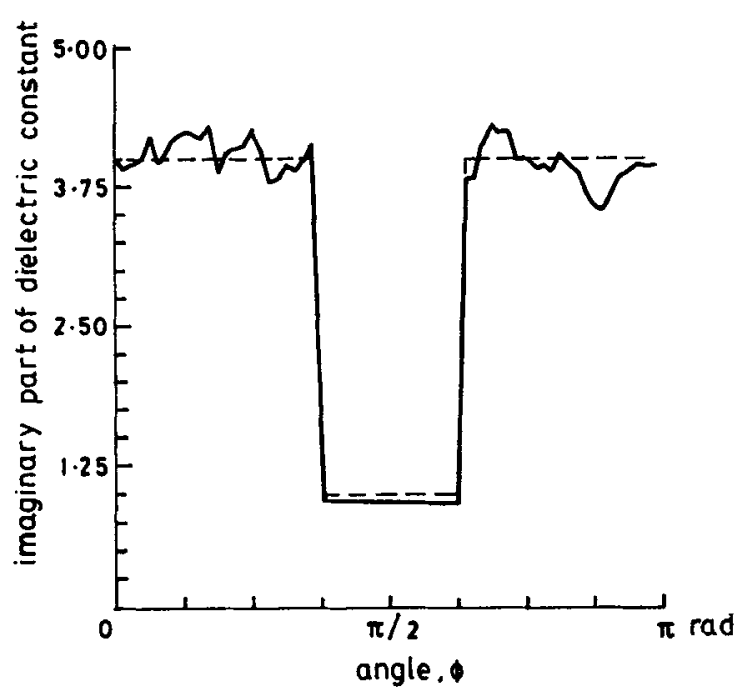

(b)

Figure 4. A comparison between the correct (indicated by - - ) and reconstructed (indicated by-_-) dielectric constants. The improved reconstruction obtained here is due to the measurement circle being kept very close to the surface of the scatterer $(R=3 \cdot 01 \hat{\lambda})$. Data corrupted with $1 \%$ Gaussian noise are used for the reconstruction. 
it comes to practical computation where we deal with a finite number of measurements that are corrupted with noise, this approach will not work.

We shall make use of the condition number defined in (13) to examine the effect of varying the radii of the measurement-circle on the sensitivity of the problem. Table 2 depicts the variations of the condition number vs. the radius $R$. It is clear from table 2 that as the radius of the measurement circle is decreased, the condition number also decreases. Thus, we expect better behaviour from the solution, when the measurements are performed close to the scatterer. The improved reconstruction in figure 4 reveals that we are indeed right.

\section{Least-squares solution}

In the previous section we have shown that the sensitivity of the shell problem can significantly be reduced by confining the measurements to the near-zone of the scatterer. We can improve the solution further by making use of additional measurement data. In this case, we shall be dealing with an $M \times N$ matrix $(M>N)$ in (2). A system of equations with more rows than columns, and with the RHS corrupted by noise, violates Hadamard's conditions, and is hence ill-posed.

We can regularize the problem by changing the definition of what is meant by an acceptable solution. In other words, the problem which is ill-posed when an exact solution is demanded, may be rendered well-posed by desiring only an approximate description of the solution. One such description is the least-squares solution to $A \mathbf{x}=\mathbf{y}$, and is given by the Moore-Penrose generalized inverse (Nashed 1976)

$$
\mathbf{x}=\left[\mathbf{A}^{\mathbf{H}} \mathbf{A}\right]^{-1} \mathbf{A}^{\mathbf{H}} \mathbf{y},
$$

where $\mathbf{A}^{\mathbf{H}}$ denotes the Hermitian conjugate of $\mathbf{A}$. The reconstructions obtained by employing the least squares solution are depicted in figure 5. Comparisons between these, and the solutions obtained earlier (figure 4) show the former to be the more accurate reconstruction. This is to be expected as the least-squares solution uses additional data and is less 'ill-posed'. In the numerical computation of the least squares solution, a $100 \%$ oversampling was employed $(N=256)$, and the data points were chosen very close to the surface of the scatterer, once again angularly equispaced, as illustrated in figure 6, to facilitate an FFT-based solution, the description of which is provided in appendix $A$.

\section{Conclusions}

In this paper, we have examined the noise-sensitivity of the shell-reconstruction problem using the concept of condition numbers and concluded that unless the measurements are confined to a zone very close to the surface of a scatterer, there is every possibility of ending up with a solution which bears very little resemblance to the correct one. Further, we have shown that by solving an overdetermined system of equations obtained by considering additional scattering data using a least-squares approach, we can improve the accuracy of the reconstruction. In appendix A, we 




(a)

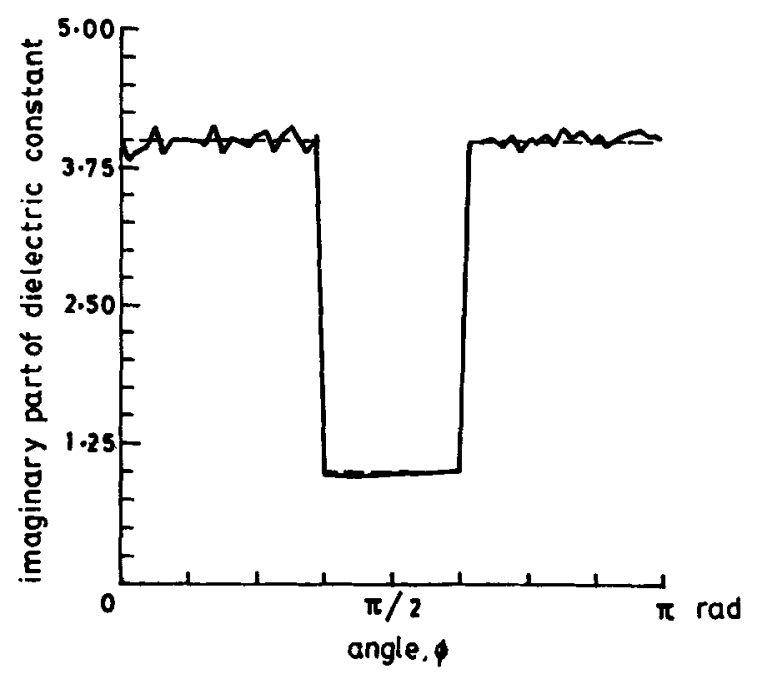

(b)
Figure 5. A comparison between the correct (indicated by ---) solution and the least squares solution (indicated by $\rightarrow$ ). The 'good' reconstruction is due to the additional data incorporated into the reconstruction procedure, and the closeness of the measurement circle to the surface of the scatterer as before.

describe an FFT-based method for implementing the least-squares solution very efficiently.

The requirement that all the measurements be made close to the surface of the scatterer is restrictive. However, it may be possible to make measurements at a convenient contour and then perform interpolation, using a scheme as the boundary element method (Kagami \& Fukai 1984), on a circle close to the shell. Whether such an interpolation can be fitted within the framework of FFT so that the computational efficiency of the solution presented here is not sacrificed, or whether it will acquit itself creditably in the presence of noise are issues that merit further investigation.

We have not made any quantitative comparisons between the theoretical error bounds and the actual errors obtained in the reconstructions, since this is against the 'philosophy' of error analysis. As Wilkinson (1971) says, 


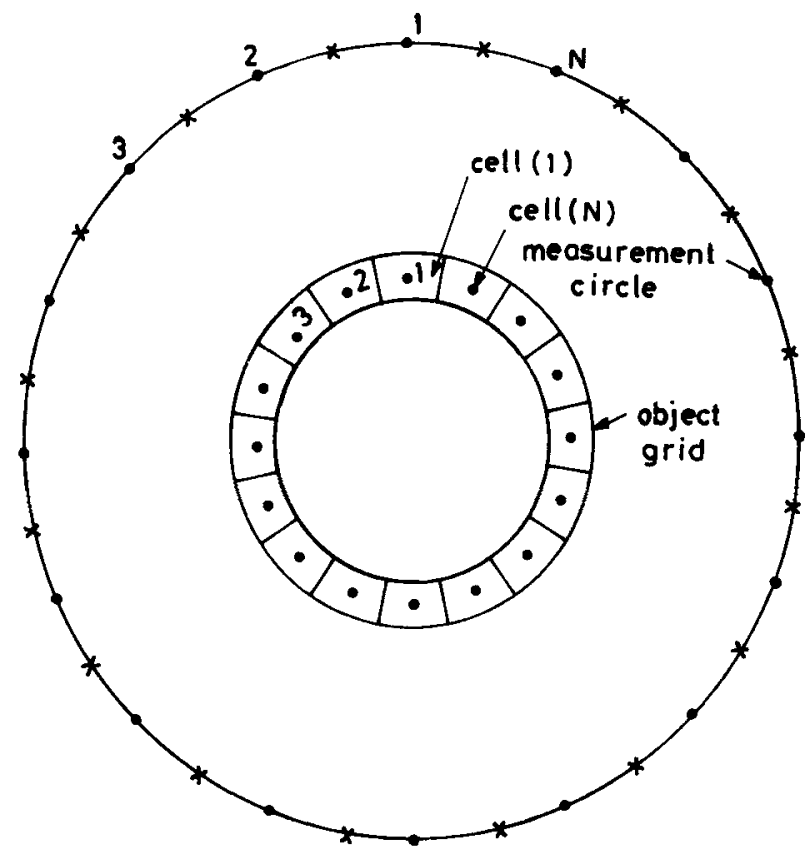

Figure 6. Measurement configuration suitable for the least squares solution. The ... indicate the locations of the original $N$ measurement points, while $x \times x$ indicate the locations of $N$ additional measurement points, with equiangular spacings.

'There is still a tendency to attach too much importance to the precise error bounds obtained by an a priori analyis. In my opinion, the bound itself is the least important part of it. The main object of such an analysis is to expose the potential instabilities, if any, of an algorithm so that hopefully from the insight thus obtained one might be led to an improved algorithm?

\section{Appendix A}

\section{A1. Some important properties of circulant matrices}

(i) Consider the evaluation of the product $\mathbf{A x}$, where $\mathbf{A}$ is an $N \times N$ matrix and $\mathbf{x}$ is an $N \times 1$ column vector. In general this will require a computational effort proportional to $N^{2}$. However, if $\mathbf{A}$ is a circulant matrix of the form,

$$
\left(\begin{array}{cccc}
a_{1} & a_{N} & \cdots & a_{2} \\
a_{2} & a_{1} & \cdots & a_{3} \\
\vdots & \vdots & \vdots & \vdots \\
a_{N} & a_{N-1} & \cdots & a_{1}
\end{array}\right),
$$

the product $\mathbf{A} x$ is the well-known cyclic convolutional sum, which can be evaluated using the FFT with a computational effort proportional to $N \log N$ (Oppenheim \& Schafer 1988, pp. 87-135).

(ii) Now, consider the evaluation of the product $\mathbf{A B}$ where both $\mathbf{A}$ and $\mathbf{B}$ are $N \times N$ matrices. This, in general, requires an effort proportional to $N^{3}$. However, if both $\mathbf{A}$ and $\mathbf{B}$ are circulant matrices, the product $\mathbf{A B}$ can be evaluated with an effort proportional to $N \log N$, as the product of $\mathbf{A}$ and the first column of vector of $\mathbf{B}$ can be cyclically rotated to get the resultant matrix. 
(iii) The inverse of a circulant matrix can be evaluated, again, with a computational complexity of $O(N \log N)$. This is seen by considering the following eigenvalue decomposition of a circulant matrix A:

$$
\mathbf{A}=\mathbf{W} \Gamma \mathbf{W}^{\mathbf{H}}
$$

where $W$ is the DFT matrix (Kay 1988, pp. 3-25), $\Gamma$ is a diagonal matrix containing the DFT coefficients of the first row of $\mathbf{A}$, and the superscript $\mathbf{H}$ denotes Hermitian conjugation. Due to the unitary nature of the DFT matrix, we can write

$$
\mathbf{A}^{-1}=\mathbf{W} \Gamma^{-1} \mathbf{W}^{\mathbf{H}}
$$

Since multiplications by the matrices $W$ and $W^{\mathbf{H}}$ can be performed using the FFT with $O(N \log N)$ complexity (Kay 1988, pp. 3-25), it is evident that $\mathbf{A}^{-1}$ can be obtained with a similar effort.

\section{A2. Evaluation of the least-squares solution}

Consider figure 6 in which the dots denote a set of $N$ measurement points which are spread equiangularly, and the crosses denote another set of $N$ measurement points which are again positioned similarly.

In $\S 2$, we have shown that the matrix $\mathbf{A}$ [see (5)] corresponding to a set of $N$ equiangularly spread measurement points is circulant. Since this is truly independent of the actual locations of the points so long as they are equiangularly spaced, it follows that the $2 N \times N$ matrix corresponding to the positions indicated by the dots and the crosses will be of the form,

$$
\mathbf{A}=\left(\begin{array}{l}
\mathbf{C} \\
\mathbf{D}
\end{array}\right),
$$

where $\mathbf{C}$ and $\mathbf{D}$ are $N \times N$ circulant matrices. From (A4) and (15) we can write the least squares solution as

where

$$
\begin{aligned}
\mathbf{x} & =\left(\mathbf{A}^{\mathbf{H}} \mathbf{A}\right)^{-1} \mathbf{A}^{\mathbf{H}} \mathbf{y} \\
& =\left(\mathbf{E C}^{\mathbf{H}} \mathbf{E D}^{\mathbf{H}}\right) \mathbf{y},
\end{aligned}
$$

$$
\mathbf{E}=\left(|\mathbf{C}|^{2}+|\mathbf{D}|^{2}\right)^{-1}
$$

Since $\mathbf{C}$ and $\mathbf{D}$ are circulant matrices, $\mathbf{C}^{\mathbf{H}}$ and $\mathbf{D}^{\mathbf{H}}$ and $|\mathbf{C}|^{2}+|\mathbf{D}|^{2}$ are also circulant matrices, and evidently the evaluation of (A6) requires an effort proportional to $O(N \log N)$.

\section{References}

Boerner W M, Vandenburg F H, Hamid M A K 1971 Determination of the electrical radius ka of a circular cylindrical scatterer from the scattered field. Can. J. Phys. 49: 804-819

Cavicchi T J, Johnson S A, O'Brien W D Jr 1988 Applications of the sinc basis moment method to the reconstruction of infinite cylinders. IEEE Trans. Ultrason., Ferroelectr. Frequency Control 35: 22-33

Datta A K, Som C 1981 On the inverse scattering problem for dielectrical cylindrical scatterers. IEEE Trans. Antennas Propag. AP-29: 392-97 
Devaney A J 1979 The inverse problem for random sources. J. Math. Phys. 20: 1687-91

Duchene B, Tabara W 1983a A geometrical optics method for assessing an inverse scattering problem for blood vessels - part I: A multistatic single frequency approach. IEEE Trans. Sonics Ultrason. 30: 13-19

Duchene B, Tabara W 1983b A geometrical optics method for assessing an inverse scattering problem for blood vessels - part 2: A monostatic multifrequency approach. IEEE Trans. Sonics Ultrason. 30: 20-26

Haykin S (ed.) 1985 Array signal processing (Englewood Cliffs, NJ: Prentice Hall) Chap. 6

Holoubek J 1976 Light scattering from unclad fibres: approximated ray theory of back-scattered light. Appl. Opt. 15: 2751-55

Jegannathan S, Ramamurthi B 1990a Scattering from a circular dielectric cylindrical shell - a fast algorithm. Electron. Lett. 27: 484-85

Jegannathan S, Ramamurthi B $1990 \mathrm{~b}$ Diffraction tomography of strongly-scattering infinite cylindrical objects of arbitrary cross-sectional shape, J. Acoust. Soc. Am. 88: 2713-2719

Johnson S A. Zhou Y, Tracey M K, Berggren M J, Stenger F 1984 Inverse scattering solutions by a sinc basis multiple source moment method - part 3. Ultrason. Imaging 6: 103-116

Kagami S, Fukai I 1984 Application of boundary-element method to electromagnetic field problems. IEEE Trans. Microwave Theory Tech. 32: 455-461

Kay S M 1988 Modern spectral estimation: Signal Processing Series (Englewood Cliffs, NJ: Prentice Hall) Chap 1

Marcuse D 1975 Light scattering from unclad fibres: ray theory. Appl. Opt. 14: 1528-32

Nashed M Z (ed.) 1976 Generalized inverse and applications (New York: Academic Press)

Oppenheim A V, Schafer R W 1988 Digital signal processing (New Delhi: Prentice Hall of India) chap. 3

Presby H M, Marcuse D 1974 Refractive index and diameter determinations of step-index optical fibres. Appl. Opt. 13: 2882-85

Richmond J H 1965 Scattering by a dielectric cylinder of arbitrary cross-section shape. IEEE Trans. Antennas Propag. 13: 334-341

Schueler C F, Lee H, Wade G 1984 Fundamentals of digital ultrasonic imaging IEEE Trans. Sonics Ultrason. SU-31: 195-216

Smith P R 1987 Exact solution of the inverse acoustic scattering problem for the one-dimensional density profile in cylindrical geometry. Ultrasonics $25: 138-140$

Sarkar T K, Weiner D D, Jain V K 1981 Some mathematical considerations in dealing with the inverse problem. IEEE Trans. Antennas Propag. AP-29(2): 373-378

Tracey M L, Johnson S A 1983a Inverse scattering solutions by a sinc basis, multiple source moment method - part I, Ultrason. Imaging 5: 361-375

Tracey M L, Johnson S A 1983 b Inverse scattering solutions by a sinc basis, multiple source moment method - part 2. Ultrason. Imaging 5: 376-393

Watkins L S 1974 Scattering from side-illuminated clad glass fibres for determination of fibre parameters. J. Opt. Soc. Am. 64: 767-772

Wilkinson J H 1971 Modern error analysis. SIAM Rev. 13: 548-568

Wilkinson J H 1983 The algebraic eigenvalue problem (Oxford: Clarendon Press) 